\title{
Vitamin U-bonded Stationary Phase in Capillary Ion Chromatography
}

\author{
Xiao-Lin JiAng, Lee Wah LIM, and Toyohide TAKEUCHI ${ }^{\dagger}$ \\ Department of Chemistry, Faculty of Engineering, Gifu University, 1-1 Yanagido, Gifu 501-1193, Japan
}

\begin{abstract}
A vitamin U-bonded stationary phase was prepared and the retention behavior of inorganic anions was examined using ion chromatography. Inorganic anions were retained on the vitamin U-bonded stationary phase under acidic as well as neutral eluent conditions in the ion-exchange mode. The elution order of the examined anions under neutral eluent conditions was nearly the same as that observed in common ion exchange mode, while the elution order observed under acidic eluent conditions was completely different from that observed in common ion exchange mode. The retention of the analyte anions under the neutral eluent conditions was due to the sulfonium groups of the vitamin U, while protonated primary amino groups caused retention of the analyte anions with different selectivity under acidic conditions. The retention factor of the analyte anions increased with decreasing eluent concentration under both eluent conditions. The present system was applied to the determination of bromide and nitrate contained in seawater.
\end{abstract}

(Received July 7, 2011; Accepted October 15, 2011; Published December 10, 2011)

\section{Introduction}

Various stationary phases have been developed in ion chromatography since its introduction in $1975 .{ }^{1}$ Most stationary phases for ion chromatography possess ion-exchange sites with charged or chargeable moieties, where ionic analytes undergo an electrostatic attractive or repulsive force.

Typical anion exchangers possess ammonium groups as the ion-exchange sites with inherent positive charge. Contrarily, the use of a cationic surfactant, e.g. cetyltrimethylammonium chloride (CTAC), simply allows hydrophobic stationary phases such as particle-packed ODS columns ${ }^{2,3}$ and monolithic ODS columns $^{4}$ as well as monolithic and packed silica columns ${ }^{5,6}$ to be applied in ion exchange chromatography to separate inorganic anions in seawater samples. Dilauryldimethylammonium bromide $^{7-10}$ was also used as the ion-interaction or the column-coating reagent to modify ODS columns and monolithic silica columns for ion chromatography. In those studies, modified monolithic silica columns provided successful separation of bromide in seawater. Tetrabutylammonium salt ${ }^{11}$ and zwitterionic surfactant (Zwittergent-3-14) ${ }^{12}$ were also used to coat ODS columns for the applications in ion chromatography.

On the other hand, weakly basic anion exchangers have been also used in ion-exchange chromatography. Depending on the $\mathrm{pH}$ of the eluent, the amino groups are protonated to work as the anion-exchange sites. For example, protonated diethylamino groups ${ }^{13,14}$ as well as protonated pyridine groups ${ }^{15,16}$ have been employed as the anion exchanger in ion chromatography.

The aforementioned anion exchangers have amino groups, typically strongly basic ammonium groups. Significant changes in retention behavior are observed using ion-pairing agents based on ammonium, phosphonium and sulfonium in liquid

† To whom correspondence should be addressed.

E-mail: take-t@gifu-u.ac.jp chromatography..$^{15}$ It is reported that tributylsulfonium provided less retention than traditional tetrabutylammonium, while tetrabutylphosphonium provided greater retention. ${ }^{17}$ Sulfonium and phosphonium anion-exchange sites have been utilized in anion exchange membranes for alkaline fuel cells. ${ }^{18}$ To the best of our knowledge, tertiary sulfonium and quaternary phosphonium groups have seldom been employed as the anion-exchange sites in ion chromatography. It can be expected that sulfonium or phosphonium-bonded stationary phases would provide different selectivity in ion chromatography.

Vitamin U ( $S$-methyl-L-methionine or (3-amino-3-carboxypropyl)-dimethyl-sulfonium) is known to possess a sulfonium group. The present research prepared vitamin U-bonded stationary phases and examined the characteristics as the anion exchanger in capillary ion chromatography.

\section{Experimental}

\section{Reagents and materials}

Reagents employed were of guaranteed reagent grade and were obtained from Wako Pure Chemical Industries (Osaka Japan), unless otherwise noted. Purified water was produced in the laboratory by using a GS-590 water distillation system (Advantec, Tokyo, Japan). All solutions used in this work were prepared using the purified water. Seawater samples were filtered through a $0.45-\mu \mathrm{m}$ membrane filter before injection. Porous 3-aminopropylsilica, TSKgel $\mathrm{NH}_{2}-60(5 \mu \mathrm{m}$ particle diameter, $60 \AA$ mean pore diameter) was taken out from the separation column commercially available from TOSOH (Tokyo, Japan)

\section{Apparatus}

The chromatographic measurements were carried out by using a capillary LC system, which was constructed by an L. TEX-8301 Micro Feeder (L. TEX Corp., Tokyo, Japan) or an 

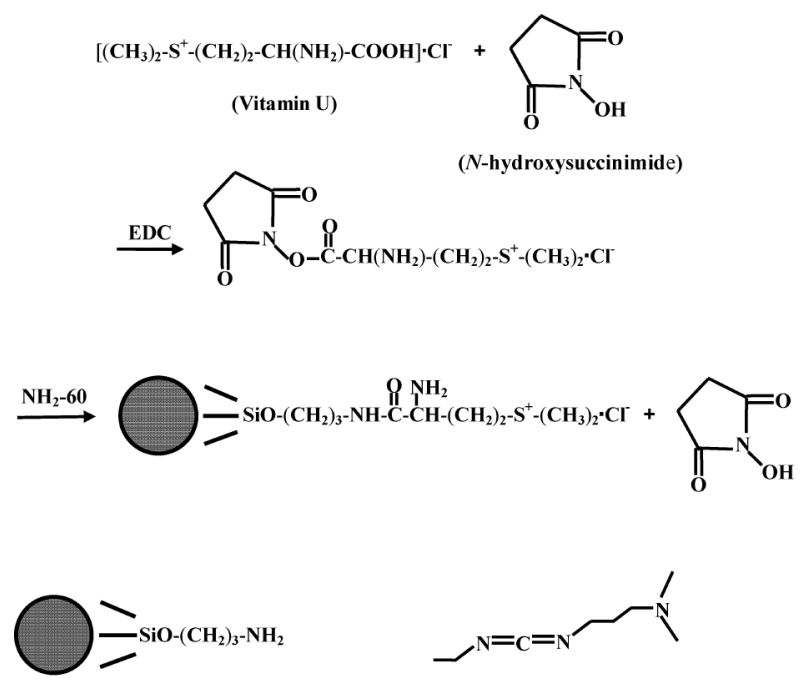

$\mathrm{NH}_{2}-60$

EDC: 1-ethyl-3-(3-dimethylaminopropyl)carbodiimide

Fig. 1 Expected reaction for the preparation of vitamin U-bonded aminopropylsilica.

MF-2 microfeeder (Azumadenki Kogyo, Tokyo, Japan) equipped with an MS-GAN 050 gas-tight syringe $(0.5 \mathrm{~mL}$; Ito, Fuji, Japan) as a pump, a Model M435 microinjection valve with an injection volume of $0.15 \mu \mathrm{L}$ (Upchurch Scientific, Oak Harbor WA) as an injector, a $100 \times 0.32 \mathrm{~mm}$ i.d. micro column, and a UV-970 UV detector (JASCO, Tokyo, Japan). The flow rate of the pump was kept at 4.0 or $4.2 \mu \mathrm{L} / \mathrm{min}$, and the UV detector was operated at $210 \mathrm{~nm}$. A capillary flow cell (75 $\mu \mathrm{m}$; JASCO) was attached to the UV detector. The data were acquired by a CAC data processor (Nippon Filcon, Tokyo, Japan).

\section{Preparation of vitamin $U$-bonded stationary phase}

A 0.22 -g amount of $S$-methyl-L-methionine $\left(\mathrm{C}_{6} \mathrm{H}_{15} \mathrm{NO}_{2} \mathrm{~S}\right.$; vitamin $\mathrm{U}), \quad 0.18 \mathrm{~g} \quad$ 1-ethyl-3-(3-dimethylaminopropyl)carbodiimide (EDC), and $0.18 \mathrm{~g} N$-hydroxysuccinimide (NHS) were placed in a $10-\mathrm{mL}$ vial. The reaction was carried out for $3 \mathrm{~h}$ at $105^{\circ} \mathrm{C}$ without any solvent. After that, a 0.25 -g amount of TSKgel $\mathrm{NH}_{2}-60$ was added into the solution and the reaction was carried out for $30 \mathrm{~h}$ at $80^{\circ} \mathrm{C}$, followed by washing with deionized water and methanol. The scheme for the expected reaction is shown in Fig. 1. The elemental analysis of the prepared stationary phases was carried out by using an MT-6 CHN Corder (Yanaco, Kyoto, Japan).

The separation column was prepared from a fused silica capillary tube $(100 \times 0.32 \mathrm{~mm}$ i.d. $)$ using a slurry packing method previously reported. ${ }^{19}$

\section{Results and Discussion}

Retention of anions on vitamin U-bonded stationary phase under neutral condition

Figure 2 illustrates the expected structure of the prepared vitamin U-bonded stationary phase. It possesses one sulfonium group and one primary amino group. The former is strongly basic, while the latter is weakly basic. This means that the former has a positive charge over a wide range of $\mathrm{pH}$, while the latter has a positive charge at lower $\mathrm{pH}$. The $\mathrm{p} K_{\mathrm{a}}$ values of the functional groups of vitamin $U$ are 11.26, 8.25 and 2.95 for sulfonium group, primary amino group and carboxyl group,
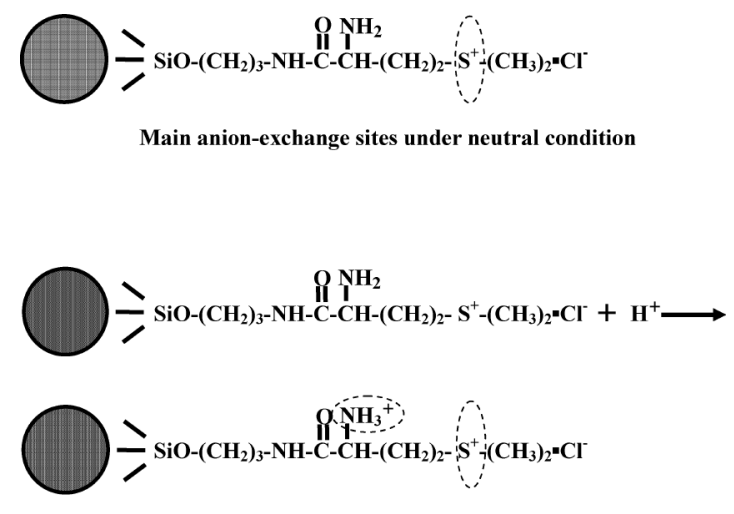

Anion-exchange sites under acidic condition

Fig. 2 Anion-exchange sites on vitamin U-bonded stationary phase under different conditions.

respectively. ${ }^{20}$ Therefore, we can expect that the vitamin U-bonded stationary phase via the carboxyl group can work as an anion exchanger in a wide range of $\mathrm{pH}$. If we operate the vitamin U-bonded stationary phase under neutral eluent conditions, the sulfonium group works for the anion-exchange sites, while both sulfonium and protonated amino groups can work as the anion-exchange sites under acidic condition. However, it should be noted that there are some residual amino groups present on the prepared stationary phase which originate from the base material. The elemental analysis results show that the concentration of the bonded vitamin $U$ groups was $0.14 \mathrm{mmol} / \mathrm{g}$, while that of the total primary amino groups was $1.10 \mathrm{mmol} / \mathrm{g}$.

Figure 3 shows the separation of an authentic mixture of UV-absorbing anions on the vitamin U-bonded stationary phase using a neutral eluent, viz., $15 \mathrm{mM}$ sodium chloride, where it is expected that sulfonium groups of the stationary phase work as the anion-exchange sites. It is seen from Fig. 3A that $\mathrm{IO}_{3}^{-}, \mathrm{Br}^{-}$, $\mathrm{NO}_{2}{ }^{-}, \mathrm{NO}_{3}{ }^{-}$and $\mathrm{SCN}^{-}$are eluted in this order. The elution order observed in Fig. 3A is slightly different from that observed in common ion chromatography: $\mathrm{IO}_{3}^{-}, \mathrm{NO}_{2}^{-}, \mathrm{Br}^{-}, \mathrm{NO}_{3}^{-}$and $\mathrm{SCN}^{-}$. This may be due to the effect of the primary amino groups on the vitamin $U$ as well as the residual amino groups. On the contrary, Fig. 3B shows the chromatogram obtained on the unmodified TSKgel $\mathrm{NH}_{2}-60$ column, showing that the retention of the anions is much smaller than that of the vitamin U-bonded column. This means that the effect of the amino groups of vitamin $U$ as well as the residual amino groups of the packing material is small under the operating conditions in Fig. 3.

The effect of the concentration of sodium chloride on the retention of the analyte anions was examined up to $20 \mathrm{mM}$ under the operating conditions as Fig. 3. It was found that the retention factor of the analyte anions decreased with increasing sodium chloride concentration. However, linear relationships between the logarithm of the retention factor and the logarithm of the sodium chloride concentration were not obtained. At higher eluent concentrations, the plots deviated from the linearity and the retention factors of analyte anions were smaller than expected.

Retention of anions on vitamin U-bonded stationary phase under acidic condition

The separation of anions under acidic condition is demonstrated for vitamin U-bonded and unmodified TSKgel $\mathrm{NH}_{2}-60$ stationary phases in Fig. 4, where a mixture of $120 \mathrm{mM}$ sodium chloride 

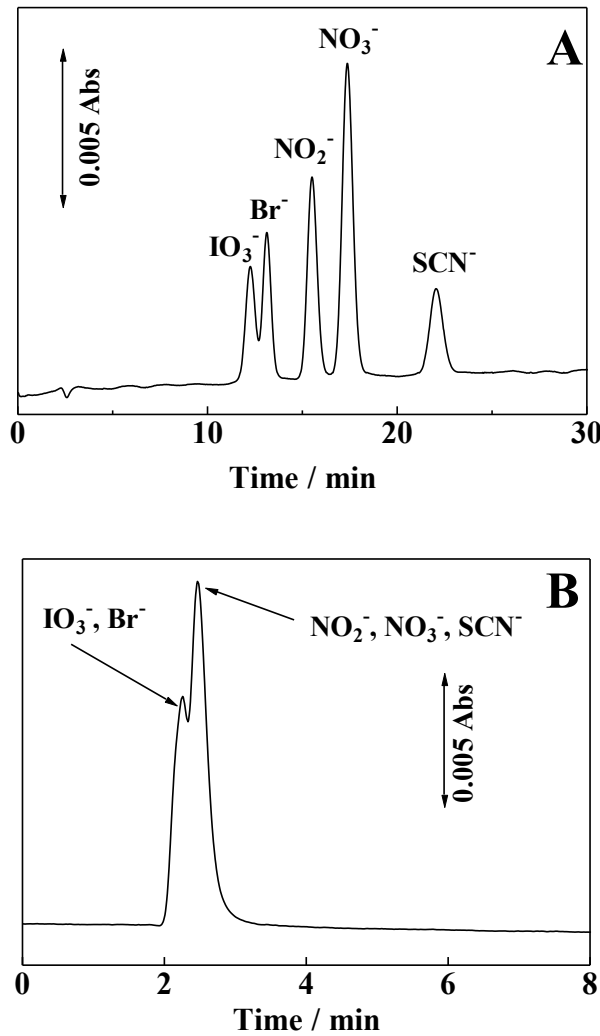

Fig. 3 Separation of UV-absorbing anions on vitamin U-bonded stationary phase (A) and unmodified TSKgel $\mathrm{NH}_{2}-60$ stationary phase (B) under neutral eluent condition. Column, vitamin U-bonded aminopropylsilica (A) and unmodified TSKgel $\mathrm{NH}_{2}-60$ (B), $100 \times 0.32 \mathrm{~mm}$ i.d.; eluent, $15 \mathrm{mM}$ sodium chloride; flow rate, $4.2 \mu \mathrm{L} / \mathrm{min}$; analyte concentration, $0.5 \mathrm{mM}$ each; injection volume, $0.15 \mu \mathrm{L}$; wavelength of UV detection, $210 \mathrm{~nm}$.

and $0.5 \mathrm{mM}$ hydrochloric acid is used as the eluent. It should be noted that the elution order observed in Fig. 4 is quite different from that observed under the neutral condition, as demonstrated in Fig. 3, and that much higher-concentration eluent is required to elute the anions in Fig. 4 compared with Fig. 3. The selectivity values of the analyte anions are nearly the same except for nitrite in Figs. 4A and 4B. It is therefore expected that the primary amino groups of the vitamin $U$ as well as the residual amino groups are protonated under the acidic condition in Fig. 4, and the analyte anions are preferentially retained on the protonated amino groups. This is the reason why the elution orders observed in Figs. 3 and 4 are totally different. The considerable increase in the retention in Fig. 4 in comparison with Fig. 3 is due to the increase in the number of ion-exchange sites under the acidic eluent condition. In other words, the total number of the amino groups is much larger than that of the vitamin $\mathrm{U}$ bonded on the aminopropylsilica. From the elemental analysis, we could estimate that $12.5 \%$ of the aminopropyl groups were modified with vitamin U and $87.5 \%$ of the aminopropyl groups were unmodified. Since the vitamin $\mathrm{U}$ possesses one primary amino group, the total number of primary amino groups present on the vitamin U-bonded aminopropylsilica is $c a .8$ times as large as the number of the vitamin $\mathrm{U}$ groups bonded on the aminopropylsilica. This is the reason why higher concentrations of the eluent should be used to elute the analyte anions under acidic conditions. In order to enhance the selectivity due to the vitamin U functional groups,
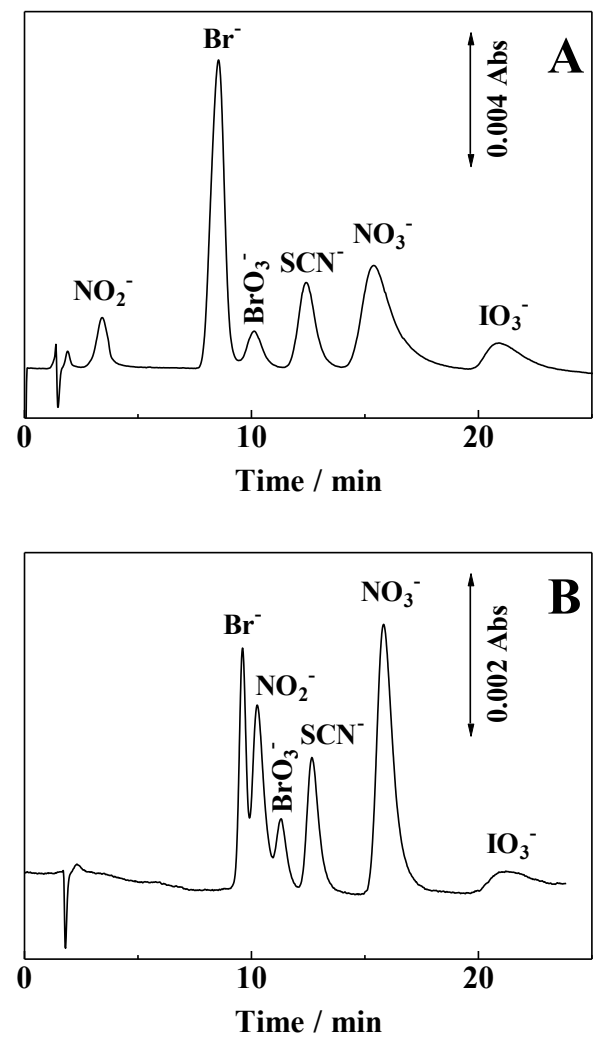

Fig. 4 Separation of UV-absorbing anions on vitamin U-bonded stationary phase (A) and unmodified TSKgel $\mathrm{NH}_{2}-60$ stationary phase (B) under acidic condition. Eluent, mixture of $120 \mathrm{mM}$ sodium chloride and $0.5 \mathrm{mM}$ hydrochloric acid; flow rate, $4.0 \mu \mathrm{L} / \mathrm{min}$; other operating conditions are the same as in Fig. 3.

one needs to increase its surface coverage. Vitamin U groups should also be chemically bonded onto supports which do not possess any ion exchangeable groups in the future work.

The effect of the concentration of sodium chloride on the retention of the analyte anions under acidic eluent conditions (in $0.5 \mathrm{mM}$ hydrochloric acid) was examined up to $400 \mathrm{mM}$. It was found that the retention factor of the analyte anions decreased with increasing sodium chloride concentration, but the plots of the logarithm of the retention factor as a function of the logarithm of the sodium chloride concentration deviated from the linearity at higher eluent concentrations. The lower retention factors observed at higher sodium chloride concentrations may be due to the increase in the ionic strength, leading to the increase in $\mathrm{pH}$ of the solution and the decrease in the ion exchange sites.

\section{Application to seawater samples}

Figure 5 demonstrates the separation of authentic mixture of five anions as well as bromide and nitrate in a seawater sample on a vitamin U-bonded aminopropylsilica column, where a mixture of $400 \mathrm{mM}$ sodium chloride and $0.5 \mathrm{mM}$ hydrochloric acid is used as the eluent. It can be seen that the retention times of bromide and nitrate for the seawater sample are the same as those for the standard sample. This indicates that the matrix ions' effects are negligible for the present separation system. This is because higher concentration of sodium chloride, viz., $400 \mathrm{mM}$, could be used as the eluent in Fig. 5 .

The repeatability of the retention time and the signal intensity were evaluated under the operating conditions in Fig. 5. 


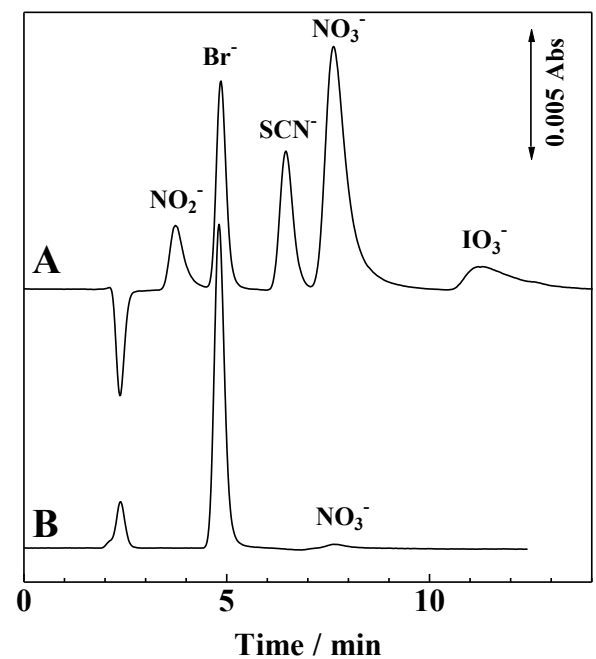

Fig. 5 Separation of anions in a seawater sample. Eluent, mixture of $400 \mathrm{mM}$ sodium chloride and $0.5 \mathrm{mM}$ hydrochloric acid; sample, standard (0.5 mM each) (A) and seawater (B); other operating conditions are the same as in Fig. 3A.

The relative standard deviations of the retention time and the peak height were $0.40-0.74$ and $1.1-1.4 \%$, respectively. Such values are acceptable for the determination of the anions. The peak height was linear to the concentration of analyte anions at least up to $0.5 \mathrm{mM}$. The detection limits of the analyte anions at signal-to-noise ratio of 3 were $1.9,0.6,0.9,0.5$, and $5.2 \mu \mathrm{M}$ for nitrite, bromide, thiocyanate, nitrate and iodate, respectively. On the other hand, the quantification limits of the analyte anions at signal-to-noise ratio of 10 were $6.7,2.0,3.0,1.7$, and $17 \mu \mathrm{M}$, respectively, under the conditions in Fig. 5. The peak height was linear for bromide and nitrate concentrations up to $0.4 \mathrm{mM}$, with correlation factors $\left(r^{2}\right)$ of 0.9996 and 0.9984 , respectively. The aforementioned detection limits achieved with the present method are comparable to or slightly worse than those reported in previous work using ion chromatography with UV detection. , $12,21-23^{2}$

According to the above validation, the concentrations of bromide and nitrate in the seawater sample were determined to be 60 and $0.12 \mathrm{mg} / \mathrm{L}$, respectively. In addition, the seawater sample was directly injected after filtration with a $0.45-\mu \mathrm{m}$ membrane. The present detection sensitivity should be improved so that trace anions such as nitrite and iodide contained in seawater could be determined. The determination results of bromide and nitrate were comparable to those we had reported. ${ }^{6}$

\section{Conclusion}

Vitamin U-bonded stationary phases prepared in this work could be used for the ion-exchange separation of inorganic anions under neutral and acidic conditions. The retention of analyte anions could be varied by changing the concentration as well as the $\mathrm{pH}$ of the eluent. The present stationary phase is expected to find novel applications due to its unique selectivity.

\section{References}

1. H. Small and T. E. Miller., Jr., Anal. Chem., 1982, 54, 462.

2. K. Ito, Y. Ariyoshi, F. Tanabiki, and H. Sunahara, Anal. Chem., 1991, 63, 273.

3. K. Ito, Y. Ariyoshi, and H. Sunahara, J. Chromatogr., 1992, 598, 237.

4. K. Ito, J. Chromatogr., A, 2005, 1083, 63.

5. A. Suzuki, L. W. Lim, T. Hiroi, and T. Takeuchi, Talanta, 2006, 70, 190.

6. X.-L. Jiang, L. W. Lim, and T. Takeuchi, Anal. Bioanal. Chem., 2009, 393, 387.

7. P. Hatsis and C. A. Lucy, Anal. Chem., 2002, 75, 995.

8. D. Connolly, D. Victory, and B. Paull, J. Sep. Sci., 2004, 27, 912.

9. D. Connolly and B. Paull, J. Chromatogr., A, 2002, 953, 299.

10. A. Suzuki, L. W. Lim, and T. Takeuchi, Anal. Sci., 2007, 23, 1081 .

11. P. Hatsis and C. A. Lucy, Analyst, 2002, 127, 451.

12. W. Hu, P. R. Haddad, K. Hasebe, K. Tanaka, P. Tong, and C. Khoo, Anal. Chem., 1999, 71, 1617.

13. M. Mori, K. Tanaka, M. I. H. Helaleh, Q. Xu, M. Ikedo, Y. Ogura, S. Sato, W. Hu, and K. Hasebe, J. Chromatogr., A, 2003, 997, 191.

14. T. Ikegami, J. Ichimaru, W. Kajiwara, N. Nagasawa, K. Hosoya, and N. Tanaka, Anal. Sci., 2007, 23, 109.

15. A. Sugii, K. Harada, and N. Ogawa, J. Chromatogr., 1986, $354,211$.

16. T. Takeuchi, T. Kawasaki, and L. W. Lim, Anal. Sci., 2010, 26, 511 .

17. C. R. Harrison, J. A. Sader, and C. A. Lucy, J. Chromatogr., A, 2006, 1113, 123.

18. G. Merle, M. Wessling, and K. Nijmeijer, J. Membr. Sci., 2011, 377, 1.

19. T. Takeuchi and D. Ishii, J. Chromatogr., 1981, 213, 25.

20. Ya. D. Fridman, S. D. Gorokhov, and L. G. Il'yasova, Koordinats. Khim., 1982, 8, 362.

21. Z. L. Chen, M. Megharaj, and R. Naidu, Chromatographia, 2007, 65, 115.

22. M. Raessler and I. Hilke, Microchim. Acta, 2006, 154, 27.

23. Y. Huang, S. F. Mou, K.-N. Liu, and J. M. Rivielo, J. Chromatogr., A, 2000, 884, 53. 